\title{
Case Report \\ Primary Intradural Extramedullary Spinal Melanoma in the Lower Thoracic Spine
}

\author{
Kathrin Hering, ${ }^{1}$ Anke Bresch, ${ }^{2}$ Donald Lobsien, ${ }^{3}$ Wolf Mueller, ${ }^{4}$ \\ Rolf-Dieter Kortmann, ${ }^{1}$ and Clemens Seidel ${ }^{1}$ \\ ${ }^{1}$ Department of Radiotherapy and Radiation Oncology, Leipzig University, Liebigstrasse 20, 04103 Leipzig, Germany \\ ${ }^{2}$ Department of Nuclear Medicine, Leipzig University, Liebigstrasse 20, 04103 Leipzig, Germany \\ ${ }^{3}$ Department of Neuroradiology, Leipzig University, Liebigstrasse 20, 04103 Leipzig, Germany \\ ${ }^{4}$ Department of Neuropathology, Leipzig University, Liebigstrasse 20, 04103 Leipzig, Germany
}

Correspondence should be addressed to Kathrin Hering; kathrin.hering@medizin.uni-leipzig.de

Received 22 January 2016; Accepted 21 March 2016

Academic Editor: Yoshiya Yamada

Copyright (C) 2016 Kathrin Hering et al. This is an open access article distributed under the Creative Commons Attribution License, which permits unrestricted use, distribution, and reproduction in any medium, provided the original work is properly cited.

Background Context. Up to date, only four cases of primary intradural extramedullary spinal cord melanoma (PIEM) have been reported. No previous reports have described a case of PIEM located in the lower thoracic spine with long-term follow-up. Purpose. Demonstrating an unusual, extremely rare case of melanoma manifestation. Study Design. Case report. Methods. We report a case of a 57-year-old female suffering from increasing lower extremity pain, left-sided paresis, and paraesthesia due to spinal cord compression caused by PIEM in the lower thoracic spine. Results. Extensive investigation excluded other possible primary melanoma sites and metastases. For spinal cord decompression, the tumor at level T12 was resected, yet incompletely. Adjuvant radiotherapy was administered two weeks after surgery. The patient was recurrence-free at 104 weeks after radiotherapy but presents with unchanged neurological symptoms. Conclusion. Primary intradural extramedullary melanoma (PIEM) is extremely rare and its clinical course is unpredictable.

\section{Introduction}

Here, we report for the first time a case of a 57-year-old female suffering from increasing lower extremity pain, leftsided paresis, and paraesthesia due to spinal cord compression caused by PIEM in the lower thoracic spine. After incomplete resection and adjuvant radiotherapy, the patient is recurrence-free at 104 weeks after radiotherapy but presents with unchanged neurological symptoms.

\section{Case Report}

A 57-year-old female patient was admitted for generalized pain in the lower extremities with mild left-sided distal paresis of the left leg and paraesthesia of the left lower leg and foot progressing for 2 months. Spinal magnetic resonance imaging (MRI) showed an intraspinal mass in the region of the lower thoracic spine at the level of the T12 vertebrae. It was hyperintense on gadolinium enhanced T1-weighted images (Figure 1(a)) and inhomogenous and hypointensive on T2weighted images (Figure 1(b)). A subsequent MRI scan of the brain and entire spine revealed no other suspect lesions. Due to increasing pain, rapid resection was planned for suspected tumor.

Intraoperatively, an intradural, black, soft, and hypervascularized neoplasm was encountered which diffusely invaded the dura. Instantaneous surgical section of the tumor confirmed a malignant melanoma. Due to diffuse tumoral infiltration and probable severe postoperative deficits the tumor was incompletely removed with decompression of the neurologic structures (Figures 1(c) and 1(d)). The macroscopic aspect of the lesion was that of a malignant pigmented tumor. Histological examination showed spinal cord tissue with an invading black tumor consisting of tight clusters of tumor cells. The cells were polygonal to spindle shaped with intracytoplasmic brown pigment. Nuclear pleomorphism 


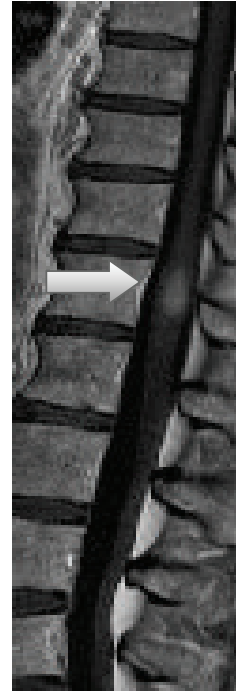

(a)

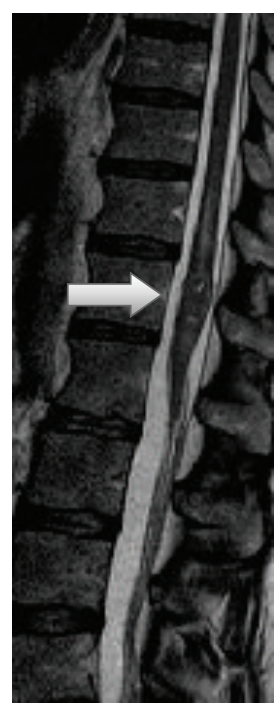

(b)

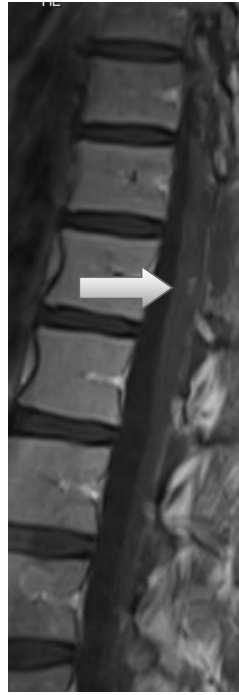

(c)

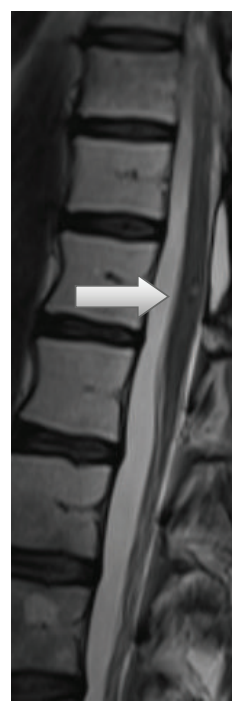

(d)

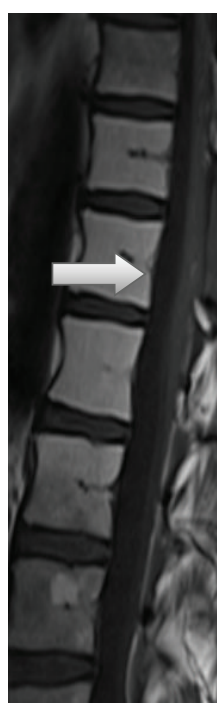

(e)

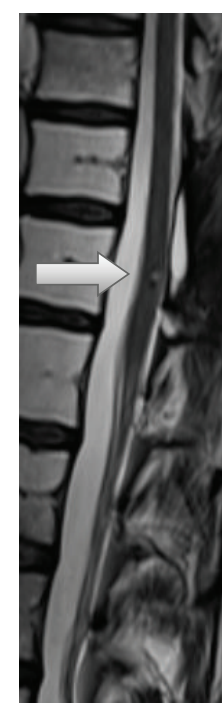

(f)

FIGURE 1: Preoperative sagittal MRI shows the spinal cord tumor at the level of the conus medullaris (arrow). T1-weighted MRI with gadolinium shows high signal intensity relative to that of the cord (a) and T2-weighted MRI shows inhomogenous hypointensive signal (b). Four weeks' follow-up sagittal T1-weighted MRI with gadolinium (c) and T2-weighted MRI (d) and 28 weeks' follow-up T1-weighted MRI with gadolinium (e) and T2-weighted MRI (f) show the residual tumor (arrow).
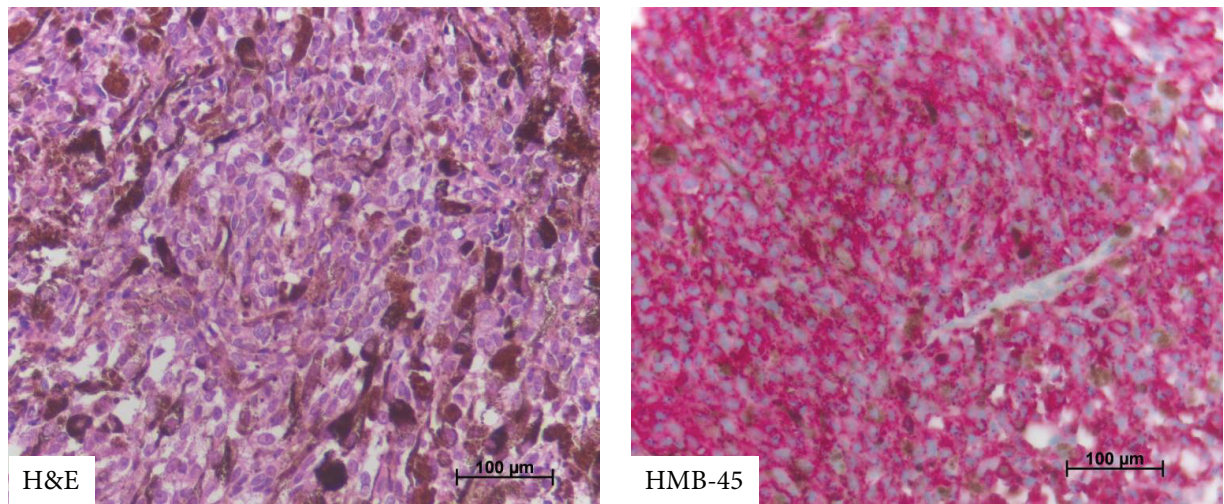

FIGURE 2: Illustration of the histology (H\&E) and immunohistochemistry (HMB-45) of the investigated tumor. Tumor cells are polygonal with prominent nucleoli and partially pigmented (H\&E). Tumor cells express the melanoma specific protein HMB-45. Tumor cells were also positive for MELAN-A and S-100 (not shown). Scale bars represent $100 \mu \mathrm{m}$; magnification $\times 400$ each.

and mitoses were visible. Immunohistochemistry showed S100 and HMB45 immunopositivity in tumor cells. They were positive for Melan-A with a mib-labeling index ranging from $5 \%$ to $10 \%$. Figure 2 illustrates histopathology and immunohistochemistry of the investigated tumor. Pyrosequencing of tumor DNA detected no BRAF V600E mutation.

The postoperative phase was uneventful and the neurological state of the patient was unchanged. Parallel to further diagnostics and two weeks after the operation, adjuvant radiotherapy was initiated. With three-dimensional conformal radiotherapy (3D-CRT) we employed a total of 40 Gray in 16 fractions in the area of T11 to L1 vertebrae with no acute toxicity except for mild nausea.

Possible primary melanoma sites and distant melanoma metastases were excluded by computed tomography (CT) scans of the chest, abdomen, and pelvis, esophagogastroduodenoscopy, coloscopy, bone scan, and dermatological, ophthalmological, and gynaecological examination. S-100 was not elevated in the serum. Finally, the diagnosis of primary intradural extramedullary spinal malignant melanoma was established. In the course of the gynaecological examination an early stage invasive ductal breast cancer of the left breast was diagnosed. Surgical treatment was offered to the patient and performed. Adjuvant chemotherapy and radiotherapy were also planned. The patient was regularly in follow-up care. At 8, 20, and 28 weeks after the operation MRI scans of the spinal cord showed a residual lesion with $6 \mathrm{~mm}$ length after 20 weeks and $4 \mathrm{~mm}$ after 28 weeks. At 15 weeks after surgery, primarily because of the newly diagnosed breast cancer, a whole body 2-deoxy-2-(18F)fluoro-D-glucose positron 
TABLE 1: Summary of primary intradural extramedullary spinal cord melanoma.

\begin{tabular}{|c|c|c|c|c|c|c|c|c|}
\hline Author & Year & Sex/age & Site & $\begin{array}{c}\text { BRAF } 600 \mathrm{E} \\
\text { mutation }\end{array}$ & Operation & Adjuvant therapy & Follow-up & Last state \\
\hline Mlaiki et al. [1] & 2004 & $\mathrm{M} / 51$ & $\mathrm{C} 5-\mathrm{C} 7$ & Unknown & Total resection & Unknown & 19 weeks & Alive \\
\hline Kounin et al. [2] & 2005 & $\mathrm{~F} / 41$ & $\mathrm{C} 2-\mathrm{C} 4$ & Unknown & Total resection & None & 12 weeks & Alive \\
\hline Kanatas et al. [3] & 2007 & $\mathrm{~F} / 76$ & C6-C7 & Unknown & Subtotal resection & RT; $30 \mathrm{~Gy}$ & 24 weeks & Alive \\
\hline Lee et al. [4] & 2010 & $\mathrm{M} / 39$ & C1-C6 & Unknown & Total resection & RT; 45 Gy & 68 weeks & Alive \\
\hline Our data & 2016 & $\mathrm{~F} / 57$ & $\mathrm{~T} 12$ & No & Subtotal resection & RT; 40 Gy & 104 weeks & Alive \\
\hline
\end{tabular}

M: male; F: female; RT: radiation therapy.

emission computed tomography (18F-FDG PET/CT) scan was performed to complete staging. Again, no local tumor progress in the spinal cord was visible. The PET/CT revealed two metabolically active skeletal lesions at the third right ridge and at the fifth thoracic vertebral body. A biopsy of both lesions showed no tumor cells. At last follow-up, 104 weeks after radiotherapy, the patient was recurrence-free and showed unchanged neurological symptoms.

\section{Discussion}

All four previously reported cases of primary intradural extramedullary spinal cord melanoma were situated in the cervical spine (Table 1). No report of PIEM in the thoracic spine has been published so far.

It is an extremely rare case, as only $1 \%$ of all melanomas located in the central nervous system (CNS) are primary melanomas $[5,6]$ and primary spinal cord melanoma is even rarer. Clinically, other, more common, differential diagnoses such as primary leptomeningeal melanomatosis, melanoma brain metastases, and primary pigmented tumor of the CNS must be taken into consideration. Our case provides a good illustration of how an extremely rare cause can underlie common neurological symptoms.

The radiological method of choice in the diagnosis of spinal cord melanoma is MRI [7]. Often a lesion in the spinal cord with hyperintensity on native T1-weighted imaging with corresponding hypointense T2 signal is shown. However, MRI findings vary according to the numbers of melanincontaining cells and haemorrhages [6]. Therefore, the distinction between differential diagnoses based solely on their MRI characteristics is difficult. The diagnosis of a melanoma can only be confirmed by histopathological examination. All reported patients with primary intradural spinal cord melanoma underwent surgery as the traditional standard treatment for primary spinal cord melanoma $[2,6]$. Due to frequent recurrence of the tumor, radical surgical resection is the first treatment of choice. The role of adjuvant therapy such as radiotherapy or chemotherapy is under debate [5].

There are no exact data for survival rates in patients with primary malignant CNS-melanoma. However, long-term follow-up has shown that primary spinal cord melanoma is generally less malignant than cutaneous melanoma metastatic to the CNS $[5,6]$.

\section{Conclusion}

Primary intradural extramedullary melanoma (PIEM) is extremely rare and its clinical course is unpredictable.

\section{Consent}

Written consent to publish was obtained.

\section{Competing Interests}

The authors declared no competing interests with respect to the research, authorship, and/or publication of this paper.

\section{Authors' Contributions}

Kathrin Hering and Clemens Seidel cared for the patient. All authors contributed to writing the report.

\section{References}

[1] A. Mlaiki, I. Ksira, M. Ladib, H. Guesmi, and H. Krifa, "Intradural and cervical primary malignant melanoma. Case report and review of the literature," Neurochirurgie, vol. 50, no. 1, pp. 42-46, 2004.

[2] G. K. Kounin, K. V. Romansky, L. D. Traykov, P. M. Shotekov, and D. Z. Stoilova, "Primary spinal melanoma with bilateral papilledema," Clinical Neurology and Neurosurgery, vol. 107, no. 6, pp. 525-527, 2005.

[3] A. N. Kanatas, M. D. Bullock, D. Pal, A. Chakrabarty, and P. Chumas, "Intradural extramedullary primary malignant melanoma radiographically mimicking a neurofibroma," British Journal of Neurosurgery, vol. 21, no. 1, pp. 39-40, 2007.

[4] C.-H. Lee, K. Y. Moon, C. K. Chung et al., "Primary intradural extramedullary melanoma of the cervical spinal cord: case report," Spine, vol. 35, no. 8, pp. E303-E307, 2010.

[5] M. S. Kim, D. H. Yoon, and D. A. Shin, "Primary spinal cord melanoma," Journal of Korean Neurosurgical Society, vol. 48, no. 2, pp. 157-161, 2010.

[6] D. Farrokh, P. Fransen, and D. Faverly, "MR-findings of a primary intramedullary malignant melanoma: case report and literature review," American Journal of Neuroradiology, vol. 22, no. 10, pp. 1864-1866, 2001.

[7] R. Sinha, T. Husain Rizvi, S. Chakraborti, C. Kumar Ballal, and A. Kumar, "Primary melanoma of the spinal cord: a case report," Journal of Clinical and Diagnostic Research, vol. 7, no. 6, pp. 1148-1149, 2013. 


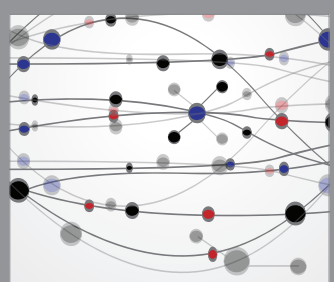

The Scientific World Journal
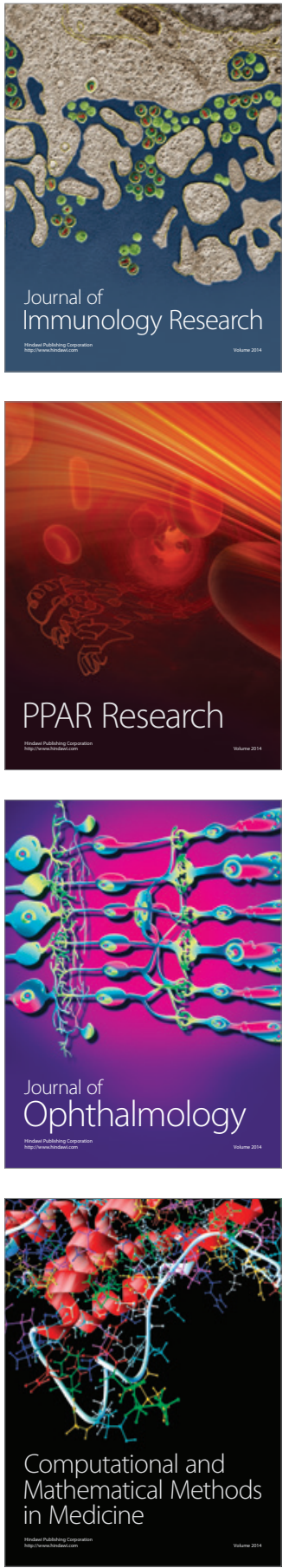

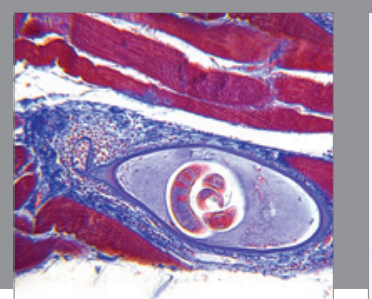

Gastroenterology Research and Practice

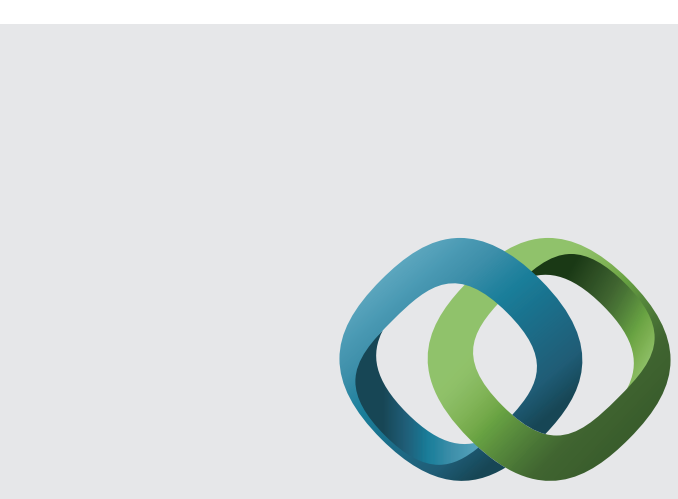

\section{Hindawi}

Submit your manuscripts at

http://www.hindawi.com
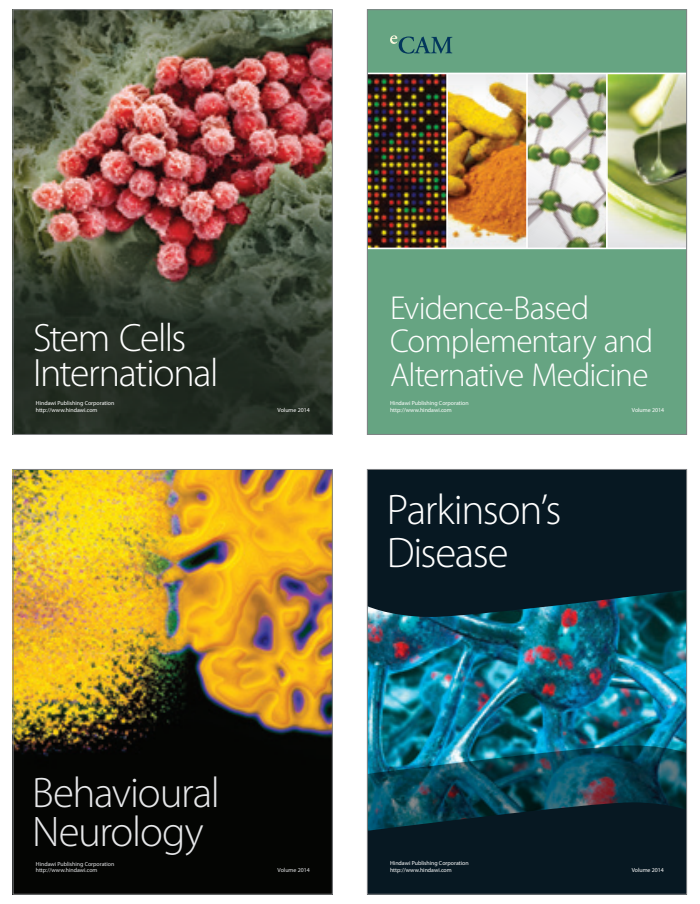
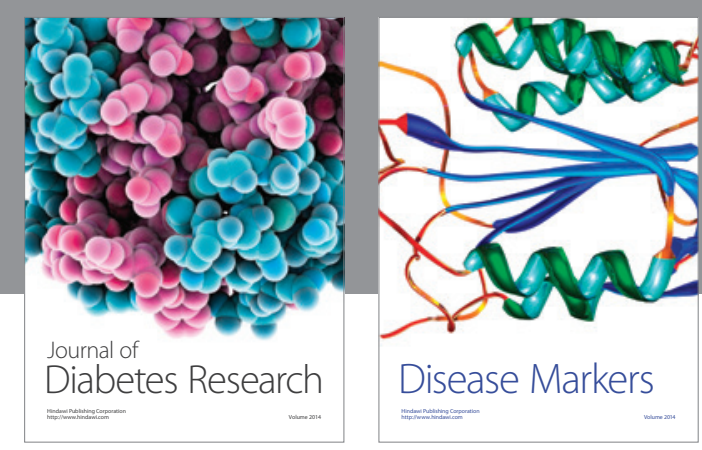

Disease Markers
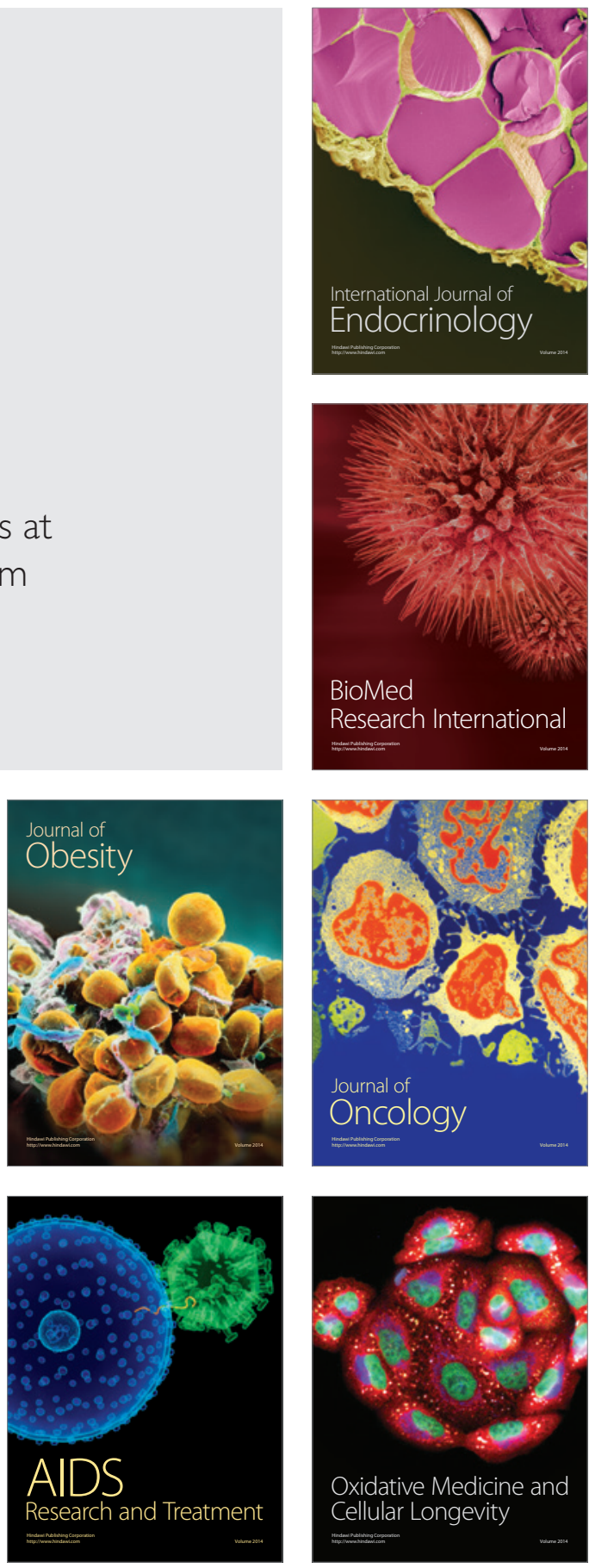